\title{
Research on Competitive Swimming Sports based on Optimal Control Theory
}

\author{
Jing meng $\operatorname{Sun}^{1}$, Yueqiu $\mathrm{Han}^{2}$ and Hui $\mathrm{Li}^{1}$ \\ ${ }^{1}$ Sports Department, Harbin Engineering University, Harbin 150000, China \\ ${ }^{2}$ International Cooperative Education College, Harbin Engineering University, \\ Harbin 150000, China \\ SunJingmeng2014@126.com \\ Abstract
}

Through the analysis of features of short-distance corpetitive swimming sports, the model of both kinetics and energy transformation is built. On the prerequisite, of a series of rational hypotheses and restrictions, the optimal analysis is performed about the distance in a given time frame with the application of the theory of optimal control. The optimal solution to propulsive force is deduced with reference to Olympic champion and experimental results by former researchers. The three-phase optimum speed distribution strategy is proposed, in good agreement with actual situations.

Keywords: Optimal control theohy, Competitive swimming, the best strategy, Olympic champion, Active drag

\section{Introduction}

Competitive swimming is a race which combines physical ability, speed and tactics. A competitor wins the chanpionship because he/she takes the lead even by a very little time lag, which, as a mater of fact, has much to do with strategies. As learnt from the Chinese history story: Tianji's horse racing, which is well-known to all and goes down in the history, it is contest of tactics. Strategy can be considered mathematical programming [1-2]. Modern mathematical methods used for competitive physical events could be traced back to the 70S of last century. In 1973, American mathematician T.B. Keller created a mathematical model to train sportsmen [3]. Middle-distance runners who were trained by his theory made remarkable achievements. Almost at the same time, Computer experts investigated throwing techniques of the then contester for world discus championship with mechanics, mathematics and computer. Proposals were raised for the improvement. So that the player achieved four meters 1onger than before in a short time. With the advantage of his scientific training method, former Soviet Union, United States and Germany all performed much better in the 1988 Seoul Olympics. Modern sports especially competitive sports have been closely associated with modern sciences, such as mathematics, mechanics, physiology and psychology. Scientific training means have been demonstrating more and more strengths. Swimming event is without exception since it's a physical movement in the water. The sports are motivated forward by swimmers' arms pull and footwork in periodical manners, low efficiency and fast consumption of energy. For the goal of maximized performance of limited physical fitness for the best result, what shall we do to accommodate velocity and thrust force reasonably in the defined race distance? That is a question of optimization and the optimal control theory is required to discuss. 


\section{Theory of Optimal Control}

The automatic control theory, evolving since the World War II, is a modern control idea which was developed based on the concept of state space. The optimal control theory is an important part to the modern control theory. Under the impetus of the development of space technology and extensive application of digital computer during 1950-1960S, the theory grew into a significant subject branch along with the rapid advancement of dynamic system optimization theory. Till today, the theory of dynamic system optimization has had lots of successful applications and been widely used in the fields like space technology, system engineering, economic management and decision, with remarkable achievements, instead of being only confined to the traditional field-automatic control. Meanwhile in the course of gradual perfection and improvement, the optimal control has produced plenty of theoretical problems to be solved. Hence, the theory is still a hot-concerned field of study [4-5].

What the optimal control theory needs to solve is: according to dynamism of the controlled target, one admissible control is selected to make the controlled run by teennjcal requirements and the specific performance index the optima. Mathematicaliy, it is a question to get the functional extremum value to meet restrictive conditions. The ontimal control theory is in its essence calculus of variation because it is only effective for non-restraints or open sets. But in practical engineering works, it is rather more a question of the best control where admissible control belongs to close set. It's necessary for us to discover new approaches and solutions to dissolve the problem. Amongst different solutions, the two are the most effective: principle of the minimum/maximum by the former Soviet scholar Pontryagin, and dynamic programming method by American scholar R. B Bellman. The minimum principle was progressively established by Pontryagin et a d after learning from Hamilton principle and strict test. It generated the classic variational theory, whichbecame a powerful instrument to deal with close-set restrictive questions. Dynamic programming method, proposed by Bellman, led to Hamilton-Jacobi theory based on the principle of optimality, which is a strategy suitable for computer calculation and handling tasks in a broader range of areas [6].

The question of optimized control is expressed by abstracting it to a mathematical model and portraying it with accurate mathematical languages. The mathematical description involves the four parts:

\subsection{State Equation of Controlled Dynamic System}

In general, before the optimal control problem is described and solved, the mathematical model of controlled system should be built, i.e. motion differential equation of dynamic system. It's necessity to abstract reasonably and simplify appropriately the issue to be concerned The law of motion-differential equation can be expressed in accordance to fundamental laws of kinetics and kinematics. Since the differential equation of the complicated controlled system cannot be written in analytic method, structure and parameter of the system is usually determined through "identification" as to create the mathematical model. No matter what way will be adopted, the controlled system of one lumped parameter can be represented by a group of first-order ordinary differential equation, state equation, like:

$$
x(t)=f[x(t), u(t), t]
$$

Where, $x(t)$ is $\mathrm{n}$-dimensional state vector; $u(t)$ is $\mathrm{m}$-dimensional controlling vector; $\mathrm{t}$ is real independent variable. It can generalize the mathematical model of controlled system with lumped parameters, such as linear time-invariant system, linear non-stationary system and linear time-varying system. 


\subsection{Target Set}

Dynamic system often shifts from one state to another for the effect of $u(t)$. For the optimal control problem, initial state is generally known, while $t_{f}$ it takes to reach the final state as well the final state $x\left(t_{f}\right)$ differs with different problems, which could be one fixed point in the state space, or a predefined range, to be more general. At large, the requirement of the final state can be expressed with the following limitations:

$$
\left\{\begin{array}{l}
g_{1}\left[x\left(t_{f}\right), t_{f}\right]=0 \\
g_{2}\left[x\left(t_{f}\right), t_{f}\right] \leq 0
\end{array}\right.
$$

The final state decides one time-variant or time-invariant collection in the state space. The collection of state which can suffice restrictions of the final state is named target set.

\subsection{Admissible Control}

Every component of controlling vector has different physical properties, like engine thrust, the deflexion angle of control piston, electromagnetic torque, volfage and electricity. They are often restrained by objective conditions, of which values can only change in a certain range. Such restraint is shown as:

Or

$$
\begin{aligned}
& 0 \leq u(t) \leq u_{\max } \\
& \left|u_{i}\right| \leqslant a,=1,2, \ldots, n
\end{aligned}
$$

Provisions of the control constraints are called the control domain, it can be written into $\Omega$. Belongs to $\Omega$ control set, it call adndission control. And recorded as $u(t) \in \Omega$.

\subsection{Performance Index}

Transferring from a ôven initial state to target set can be achieved through different controls. In order to-measure the effect of the control system on each control function, it's required to build performance index function, renamed performance function, which can be employed to evaluate the gogdness/badness of control effect or quality. Items and forms of such index are different along with specific problems. Even if the problem is same, the index will be different owing 10 different point of view. In some cases, some require time optimization; some require fuel consumed the least; some require the both. But whatever the problem is, the function can be summarized into a general form as follows:

$$
J[u(\square)]=S\left(x\left(t_{f}\right), t_{f}\right)+\int_{t_{0}}^{t_{f}} L(x, u, t) d t
$$

Which, the first part on the equation right is final-value performance index and the second is integral performance index; those having both combined are composite performance indices. What the problem of optimization has to solve is optimize such indices.

Looking back at the description of optimization problem above, we can get the expression: known controlled system state equation and the specified initial state.

$$
\begin{gathered}
x(t)=f[x(t), u(t), t] \\
x\left(t_{0}\right)=x_{0}
\end{gathered}
$$

Admissible control $u(t) \in \Omega, t \in\left[t_{0}, t_{f}\right]$

We introduced briefly the theory of optimal control as well as mathematical description and general expression of optimized problem. Next, we'll discuss the problem of the 
optimized control of time in short-range competitive swimming in virtue of the aforesaid theory.

\section{Analysis of Optimization of Short-distance Competitive Swimming Sports}

Over many years, competitive swimming has developed into an important event in today's physical culture. For the improvement of swimming performance, ideas experienced many innovations in the world, which related mainly to increasing technological quality of training, making swimming skills and training methods be better and more reasonable. That will not only maximize players' physical potentials and also help them use various tactics to progressively make better achievements. For the purpose of strategies, the pape applies the optimal control theory to study the competitive event by converting equivalently the problem of getting minimum time in a given route to that of obtaining the longest distance. Through theoretical inference, the optimal solution is acquired in certain conditions, how to distribute reasonably speed and stamina for the longest way that a wimmer can reach in given time range.

\subsection{Mathematical Model}

3.1.1. Kinetic Model: Human body is reduced to a particle and only the horizontal movement is considered. According to Newton's second,law:

$$
m v(t)=f(t) \frac{1}{2} C_{d} A \rho V^{2}(t)
$$

Where, $F(t)$ is equivalent horizontal thrustforce; $\mathrm{m}$ is quality of the player; $\rho$ is density of water; $C_{d}$ is resistance coefficient; A is sectional area of body's horizontal projection; $\mathrm{V}$ is swimming speed. As the thrust force is limited, we set maximum value Q. $F(t)$ is normalized to $u(t)$ :

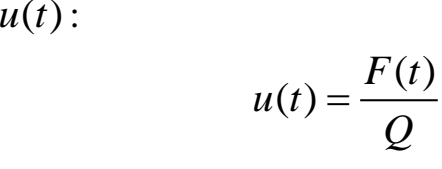

Therefore, $0 \leq u(t)$ 1. Considering A \& Q is different from people [7], but they are linearly bound with $\mathrm{m}$, so quality unit is converted into the following two factors:

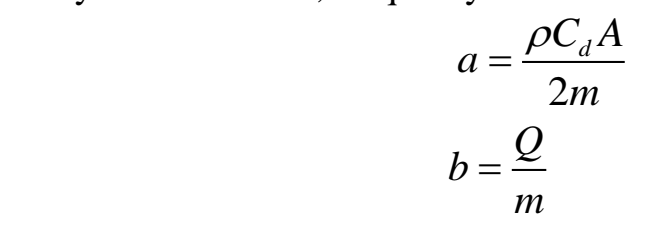

The kinetic equation is simplified into

$$
V(t)=-a V^{2}(t)+b u(t)
$$

3.1.2. Energy Transformation Model: Swimming is a complicated physiological process. Human body relies on aerobic and anaerobic metabolism to furnish energies for physical exercise [8]. The change rate of energy storage $E(t)$ can be shown like:

$$
E(t)=K-P-N-R
$$

where, $\mathrm{K}$ is power for generating calories through aerobic and anaerobic metabolism; $p=b u(t) V(t)$ is horizontal thrust power; $\mathrm{N}$ is non-trust power, which enables water of a 
certain quality with kinetic energy; $\mathrm{R}$ is wasted power in the form of thermal energy. Wasted power of heat energy and non-thrust power bear complicated non-linear relationship with moving speed and time. It's reported that in the 50m-long swimming, anaerobic metabolism is $95 \%$ and aerobic metabolism $5 \%$; in the $100 \mathrm{~m}$-long competition, anaerobic metabolism is $80 \%$ and aerobic $20 \%$. It's obvious that the longer the distance is, the lower percentage anaerobic metabolism has and the higher aerobic does. Thus, in the paper, the model is designed for short-distance competitive swimming. We assume in the short competition, body's power is all provided by aerobic metabolism, disregard of heat dissipation and nonthrust force. Then we have:

$$
E(t)=-b \cdot u(t) \cdot V(t)
$$

\subsection{Expression of the Optimized Question}

In fact, in the competitive swimming, what's concerned is the shortest time in given distance. For the purpose of convenience, we analyze its equivalence problem, i.e. about how to distribute speed and physical ability in a given time to swim across the longest distance. For unification, we assume all swimmers start from the pool wall and the initial speed is 0 ; also their turn-back movements are neglected. The optinal problem is:

Non-linear time-invariant systems known equation is $x=f$, where

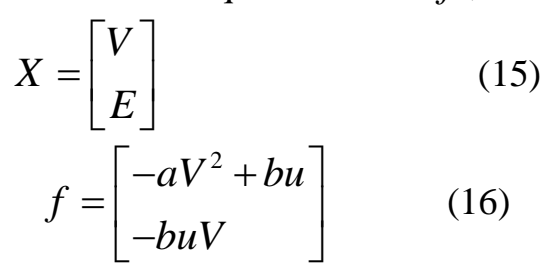

Permit propulsion $u(t), \quad \leq u(t) \leq$ make the system from a given initial state start to reach the final state of a given moment $t_{f}$, so as to meet state constraints

$$
\left.X(t)\right|_{t=0}=X_{0}=\left[\begin{array}{l}
0 \\
E_{0}
\end{array}\right]
$$

\subsection{Theoretical Analysis and Solution}

For the state equation, the right part refers to non-linear function. So far no generic way of discrimination has been found for the optimal control. But it's believed the best solution exists in terms of physical meaning of its extreme problem [9]. Performance indices are transformed to the equivalent ones with Lagrangian multiplier method and Kuhn-Tucker theorem.

$$
J[u(\square)]=-\beta E\left(t_{f}\right)+\int_{0}^{t_{f}}\left[-V+\bar{\lambda}^{T}(t)(f-X)\right] d t
$$

Where, $\bar{\lambda}(t)=\left[\lambda_{1}(t), \lambda_{2}(t)\right]^{T}$ is non-zero state vector $\beta$ is constant, and to $\beta$ meet

$$
\beta \geq 0, \beta E\left(t_{f}\right)=0
$$

Structure of Hamilton function $\mathrm{H}$ as follows:

$$
H(X, \bar{\lambda}, u)=-V+\bar{\lambda}^{T} \cdot f=-V+\lambda_{1} \cdot\left(-a V^{2}+b u\right)+\lambda_{2} \cdot(-b u V)
$$

Applying the principle of pontryagin minima, it can be obtained:

Association equation of state: 


$$
\left\{\begin{array}{l}
\lambda_{1}=\frac{\partial H}{\partial V}=1+2 a \lambda_{1} V+\lambda_{2} b u \\
\lambda_{2}=\frac{\partial H}{\partial E}=0
\end{array}\right.
$$

Cross-sectional condition:

$$
\left\{\begin{array}{l}
\lambda_{1}\left(t_{f}\right)=\frac{\partial S\left(X\left(t_{f}\right)\right)}{\partial V\left(t_{f}\right)}=0 \\
\lambda_{2}\left(t_{f}\right)=\frac{\partial S\left(X\left(t_{f}\right)\right)}{\partial E\left(t_{f}\right)}=-\beta
\end{array}\right.
$$

Hamilton function $\mathrm{H}$ as $u(t)$ unction, take the absoluterminimum when $u(t)=u^{*}(t)$

$$
\left.H\left(x^{*}(t), \bar{\lambda}(t), u^{*}(t)\right)\right)=\underset{0 \leq u(t) \leq 1}{\operatorname{MIN}} H\left(x^{*}(t), \lambda\left(t^{\prime}\right), u^{*}(t)\right)
$$

Hamilton function $\mathrm{H}$ rail line is at a constant alue in optimal:

$$
\left.H\left(x^{*}(t), \bar{\lambda}(t), u^{*}(t)\right)\right)=H\left(x\left(t_{f}\right), \bar{\lambda}\left(t_{f}\right), u\left(t_{f}\right)\right)=\text { const }
$$

By the formula (21), (22) to

$$
\begin{gathered}
\lambda_{2}(t)=-\beta \\
\lambda_{1}(t)=1+2 a \lambda_{1} V-\beta b u
\end{gathered}
$$

Thus Hamilton function becomes:

$$
H(\dot{X}, \hat{\imath}, u)=-V, \vec{a} \lambda_{1} V^{2}+\left(\lambda_{1}+\beta V\right) b u
$$

By equation 23, in other quantity is unchanged, u t should make $\mathrm{H}$ take the absolute minimum, so:

$$
u^{*}(x)=\left\{\begin{array}{lc}
1, & \lambda_{1}+\beta V<0 \\
0, & \lambda_{1}+\beta V>0 \\
\text { uncertain }, \lambda_{1}+\beta V=0
\end{array}\right.
$$

Where, uncertain does not mean the absence of the optimal solution but that the best solution can be achieved only from extremum conditions, any value in $0 \leq u^{*}(t) \leq 1$ is acceptable.

When the time of a non-zero distance exists $\left(t_{i}, t_{j}\right) \subset\left[0, t_{f}\right]$ and $\lambda_{1}+\beta V=0$, the optimal solution is singular; otherwise it is normal. At this moment, the optimal solution is timehopping function of 0,1 . When it's singular, we take the derivative of $\lambda_{1}+\beta V=0$ to get the optimal solution by formula (12) and (21).

$$
1-3 a \beta V^{2}=0 \quad t \in\left(t_{i}, t_{j}\right)
$$

Therefore, only when $\beta>0$, the above type can be established, and:

$$
V(t)=\sqrt{\frac{1}{3 a \beta}} \quad t \in\left(t_{i}, t_{j}\right)
$$




$$
u(t)=\frac{1}{3 b \beta} \quad t \in\left(t_{i}, t_{j}\right)
$$

Therefore, only when $\beta>0$ the upper formula can exist singular situation, and $\beta=0$ can exist normal circumstances exist, if the singular case, the singular time interval speed and propulsion are literal

\section{Experiment Design and Discussion}

With experimental measuring results of dynamic resistance in the men s free-style swimming, we can have $a=0.32\left(\mathrm{~m}^{-1}\right)$. At one Olympic Games, the champion record of men's 50/100/200m-distance freestyle swimming is respectivê 1 y 21.98s, 48.30s, $105.35 \mathrm{~s}$. Since $50 \mathrm{~m}$ is the shortest range, it can be assumed $50 \mathrm{~m}$ that related players moved forward from the best propulsive force. We mark $50 \mathrm{~m}$ championship record for $T_{50}, 100 \mathrm{~m}$ championship record for $T_{100}$. In another way when $t_{f}=T_{50}$, the optimal solution is 1 and:

$$
\int_{0}^{T 50} \frac{\exp (r t)-1}{\exp (r t)+1} V d t=50
$$

The $T_{50}=21$ substituting into the formula 32 can be obtained:

$$
\begin{gathered}
V_{e}=\sqrt{b+a} \subseteq 2.37(\mathrm{~m} / \mathrm{s}) \\
b=a V_{e}^{2}=1.79(\mathrm{~N} / \mathrm{Kg}) \\
r=2 \sqrt{a b}=1.52(1 / \mathrm{s})
\end{gathered}
$$

At the end of time energy shound be positive, substitution formula 31 can be obtained:

$$
E_{0}<201.0(\mathrm{~J} / \mathrm{Kg})
$$

If the advance in $100 \mathrm{~m}$ and above, in the last moment when energy is negative, the $t_{f}=T_{100}=48.3$ substituting into the formula 32 can be obtained:

$$
E_{0}<201.9(\mathrm{~J} / \mathrm{Kg})
$$

The E T the interval [89.9,201.9] in step of 1 is gradually increasing the numerical solution, using a least squares fit $100 \mathrm{~m}, 200 \mathrm{~m}$ race, and the 10 feasible solutions are compared to determine the optimal solution $0\left\{1, u_{c}, 0\right\}$, with the best energy value:

$$
E^{*}{ }_{0}=176(\mathrm{~J} / \mathrm{Kg})
$$

Using the numerical parameters have been obtained, it calculates the maximum distance corresponding, when given $t_{f}=48.3 \mathrm{~s} t_{f}=105.35 \mathrm{~s}$. Comparison of the relative error, as shown in Table 1 
Table 1. Comparison of the Theoretical Corresponding Distance and Actual Distance

\begin{tabular}{|c|c|c|c|}
\hline$t_{f}(s)$ & actual distance & Theoretical optimal solution & relative error \\
\hline 21.98 & 50 & 50.00 & 0 \\
\hline 48.3 & 100 & 107.55 & $7.55 \%$ \\
\hline 105.35 & 200 & 182.01 & $-8.88 \%$ \\
\hline Using & $E_{0}^{*}$ can calculate the critical moment $T_{c}=42.23(s)$, when
\end{tabular}

given $t_{f}=T_{c}=42.23(\mathrm{~s})$, it can obtain the maximum distance: $D_{\max }=98.32(\mathrm{~m})$

When given $t_{f}>T_{c}=42.23(s)$, the optimal solution is $\left\{1, u_{c}, 0\right\}$, the propulsion force and velocity distribution is:

$$
\begin{gathered}
u^{*}=\left\{\begin{array}{l}
1, \quad t \in\left[0, t_{1}^{-}\right] \\
u_{c}, t \in\left[t_{1}^{+}, t_{2}^{1}\right] \\
0, t \in\left[t_{2}^{+}, t_{f}\right]
\end{array}\right. \\
V^{*}=\left\{\begin{array}{l}
\frac{2.37 \exp (1.52 t)-2.37}{V_{c}, t \in\left[t_{1}, t_{2}\right]} \\
\frac{\exp (1.52 t)+1}{V_{c}}, t \in\left[0, t_{1}\right]
\end{array}\right.
\end{gathered}
$$

Where

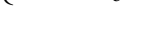

$$
\frac{2.37 \exp \left(1.52 t_{1}\right)-2.37}{\exp \left(1.52 t_{1}\right)+1}
$$

The optimal time $t_{1}^{*} t_{2}^{*}$ is afunction of $t_{f}$, but the formula is very complex, Figure 1 shows the corresponding $t_{1}^{*} t_{2}^{*}$ Numerical solution curve at the time $[44,105]$ changes
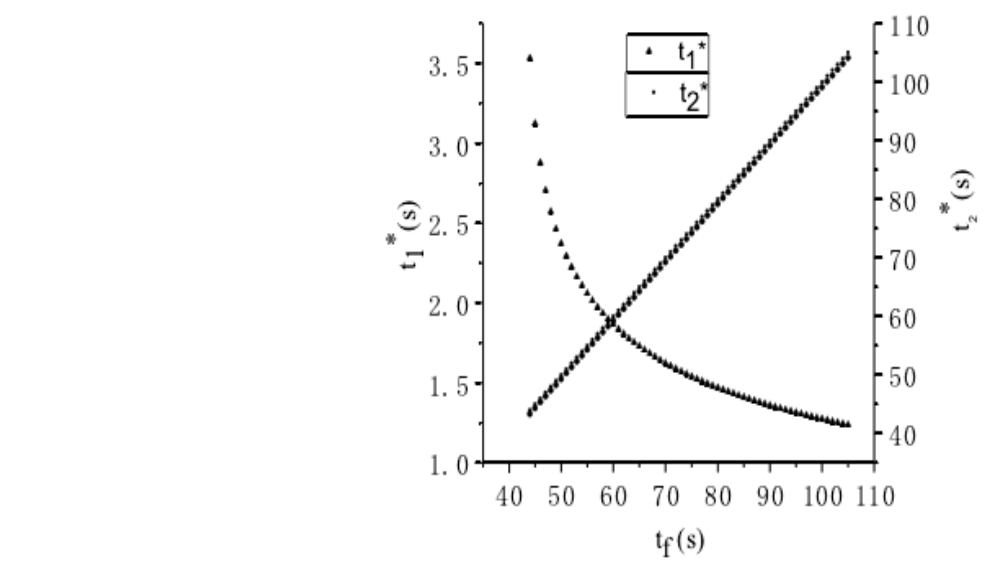

Figure 1. When $t_{f}$ is changed, the Numerical Solution of the corresponding

$$
t_{1}^{*} t_{2}^{*}
$$


In the case of $t_{f}=48.3 \mathrm{~s}$ can obtain the optimal propulsion and optimum speed curve as shown in Figure 2 and Figure3, which may have:

The first transition time $t_{1}^{*}=2.54 \mathrm{~s}$, the second transition time $t_{2}^{*}=47.61 \mathrm{~s}$, uniformspeed is $2.27 \mathrm{~m} / \mathrm{s}$, propulsion $u_{c}=0.92$

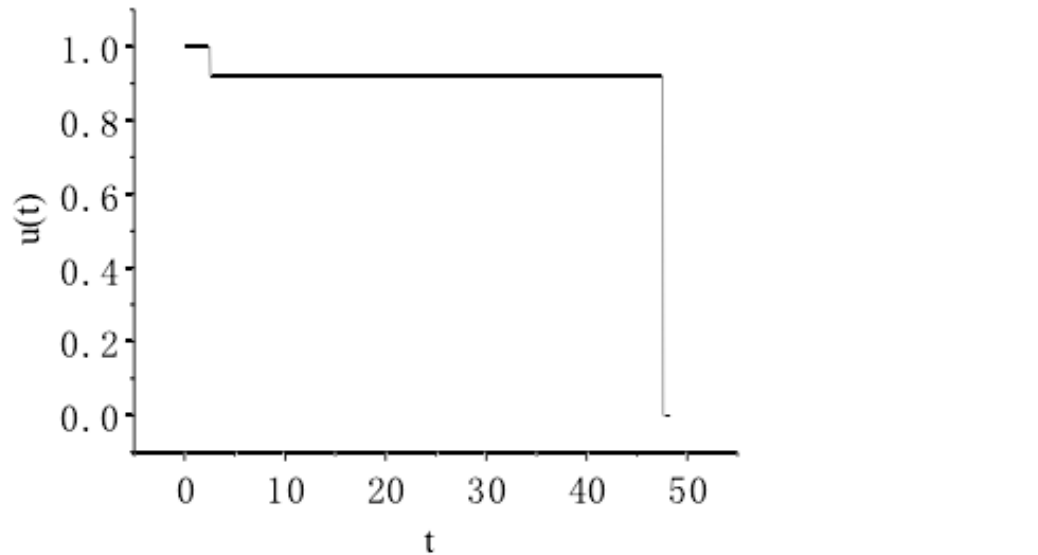

Figure 2. When given $t_{f}=48.35$ Promoting Optimal Propulsion Distribution

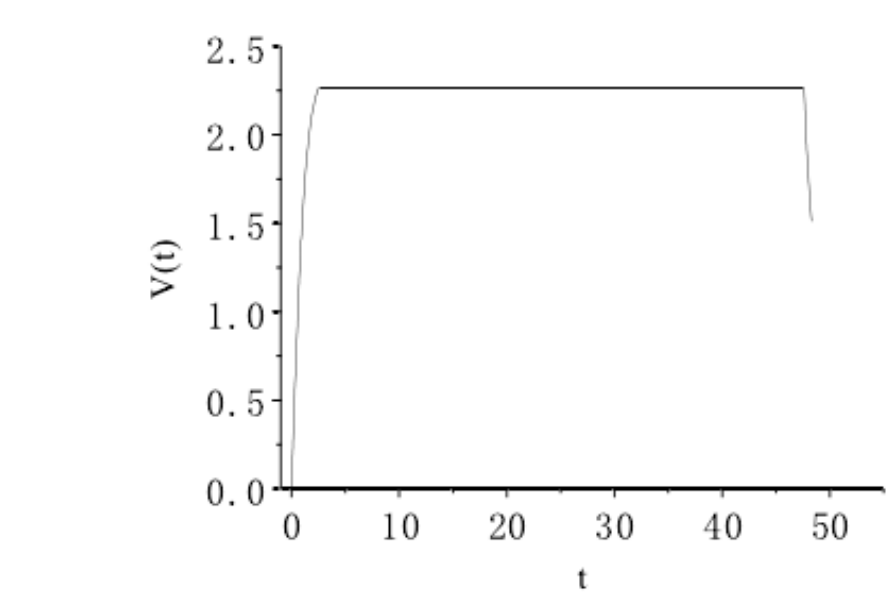

Figure 3. When Given $t_{f}=48.3 \mathrm{~s}$ Optimal Velocity Distribution

We can see the optimal solution $\left\{1, u_{c}, 0\right\}$ is a three-phase strategy, which divides the whole time range $\left[0, t_{f}\right]$ into three phases. The first stage means normalized driving force is constantly the maximum value 1 , with speed accelerating rapidly from 0 . Then at point $t_{1}$, the force changes to a steady value $u_{c}$ and now it's the second phase. The speed stays steady accordingly. In the first two phases, human's stored energy consumes away till all energy $E_{0}$ consumes up and the time jumps to point $t_{2}$. The propelling force changes to 0 . Finally in the third period, speed slows down. Contestants begin to sprint with the speed acquired before. 
Yet, when they're spurting, their speeds are decelerating and that they can reach the longest distance in the given time. As seen, when $t_{f}=48.3 \mathrm{~s}$, in the first and third phase, time consumes less while the second phase takes the most time, which is mostly used for uniform movement.

In the last phase of the optimal solution $\left\{1, u_{c}, 0\right\}$, speed is decreasing, which is beyond our understanding. But it's confirmed in real life. According to on-site observation and data statistics about swimming competition in the National Games, we find that of competitors ranking the first three positions in single $100 / 200 \mathrm{~m}$ event, $50 \%$ chose to slow down, moving slower in the latter-range than the former. Besides, we presume human's stored energy $E_{0}(t)$ is all consumed for horizontal thrust, but in reality, only a very small portion is converted to the force. It means effective output is too low, less than $10 \%$. But it does notprevent us from the supposition that $E(t)$ is the energy to be really used by human body, One more thing to stress is when we were obtaining the best solution and fittng E, 50/100/200m freestyle swimming records were used. Actually three records were enough to get the optimal solution in the case of limited time hops of driving force, and the shortest the distance is, the more precise $\mathrm{E}$ is and the distribution of driving force and speed conform better with real situation, because the model is created for short distance Ket, the optima solution remains $\left\{1, u_{c}, 0\right\}$, what is different is $t_{1}^{*}$ and $t_{2}^{*}$. Although $\{1, \mu, 0\}$ is deferred in the short distance, it coincides basically with real situation, significant folong and hedium distance race, because in the race, participants make uniform movement, which will help save energy a lot and make much more scores.

\section{Conclusion}

This paper analyzes the problem is equivalent to the maximum distance, it establish a model of energy conversion is onty suitable for short-distance situation, because in long distance-based human respiratory aerobic respiration, which is bound body to absorb aerobic respiration part of the extra energy, at this time if the same method is also used to analyze the model must be corrected.

A model was created to describe the dynamics and energy conversion in short distance competitive swimming Based on some hypotheses and constraints, the study made an optimization analysis of the distance traveled in a given time with optimal control theory. Using the records of the Olympics games and the results from previous research, an optimal solution of propulsion was derived. Correspondingly, the optimal three stage tactic for velocity distribution was put forward, which was in agreement with the practice.

\section{References}

[1] W. Gang, "The Olympic Games swimming team defeat Chinese multivariate analysis", Journal of Guangzhou Sports University, vol. 21, no. 2, (2008), pp. 71-73.

[2] L. Ma and L. Fu, "The theory of optimal control for nonlinear system. Science and technology information", vol. 19, (2010), pp. 451-502.

[3] J. Chen, Y. Cao and A. Zou, "Research on the optimal control theory and its application in the engineering", Science and technology information, vol. 23, (2010), pp. 113-114.

[4] H. Guiji, "Development and Prospect of optimal control theory", Journal of Shanghai Dian Ji University, vol. 03, (2005), pp. 33-35.

[5] W. Li, X. Liang and Q. long, "Development and Prospect of optimal control theory", Guangdong automation and information engineering, vol. 01, (2003), pp. 1-4. 
[6] H. Yang, "Study on the application of optimal control theory in power system automation", The electronic world, vol. 22, (2013), pp. 83-84.

[7] R. A. Brandt, and M. A. Pichowsky, "Conservation of energy in competitive swimming", J. Biomechanics, vol. 28, (1995), pp. 925-933.

[8] H. Behncke, and Osnabrück, "Optimization models for the force and energy in competitive sports", Mathematical Methods in the Applied Sciences, vol. 9, (1987), pp. 298-311.

[9] H. Li and L. Li, "Discussion on several problems of training theory and application of swimming energy", Swimming, vol. 3, (2011), pp.11-14

\section{Author}

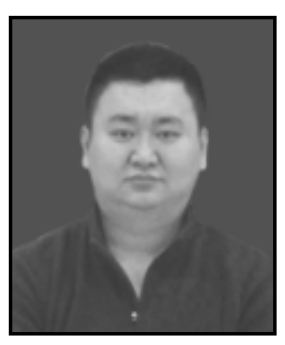

Sun Jing-meng, he is an Associate Professor at Sports Department of Harbin Engineering University, he is Master in Ideological and Political Education in Harbin Engineering University in $2005 . \mathrm{He}$ is in the research of sports teaching and training.

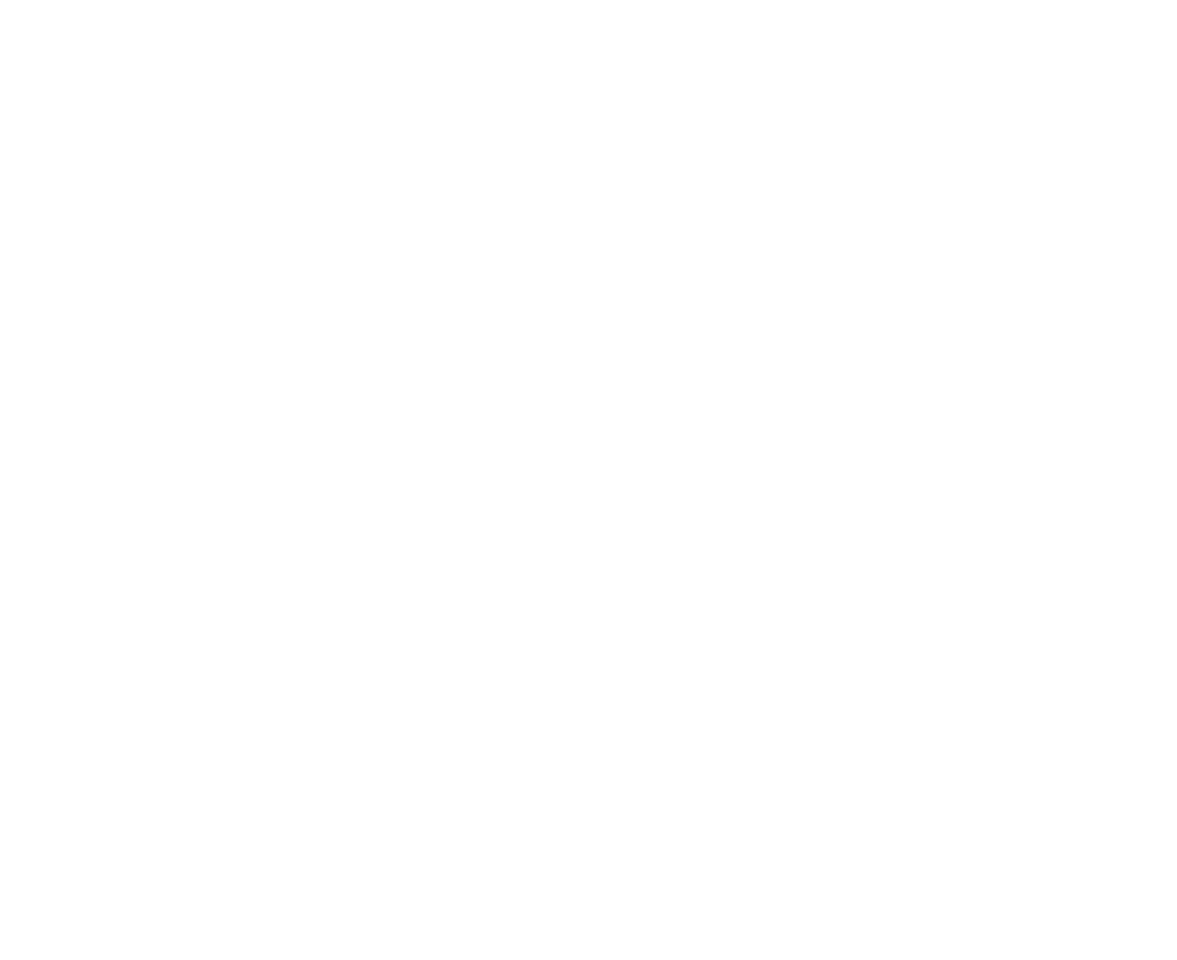


International Journal of Multimedia and Ubiquitous Engineering Vol.9, No.9 (2014)

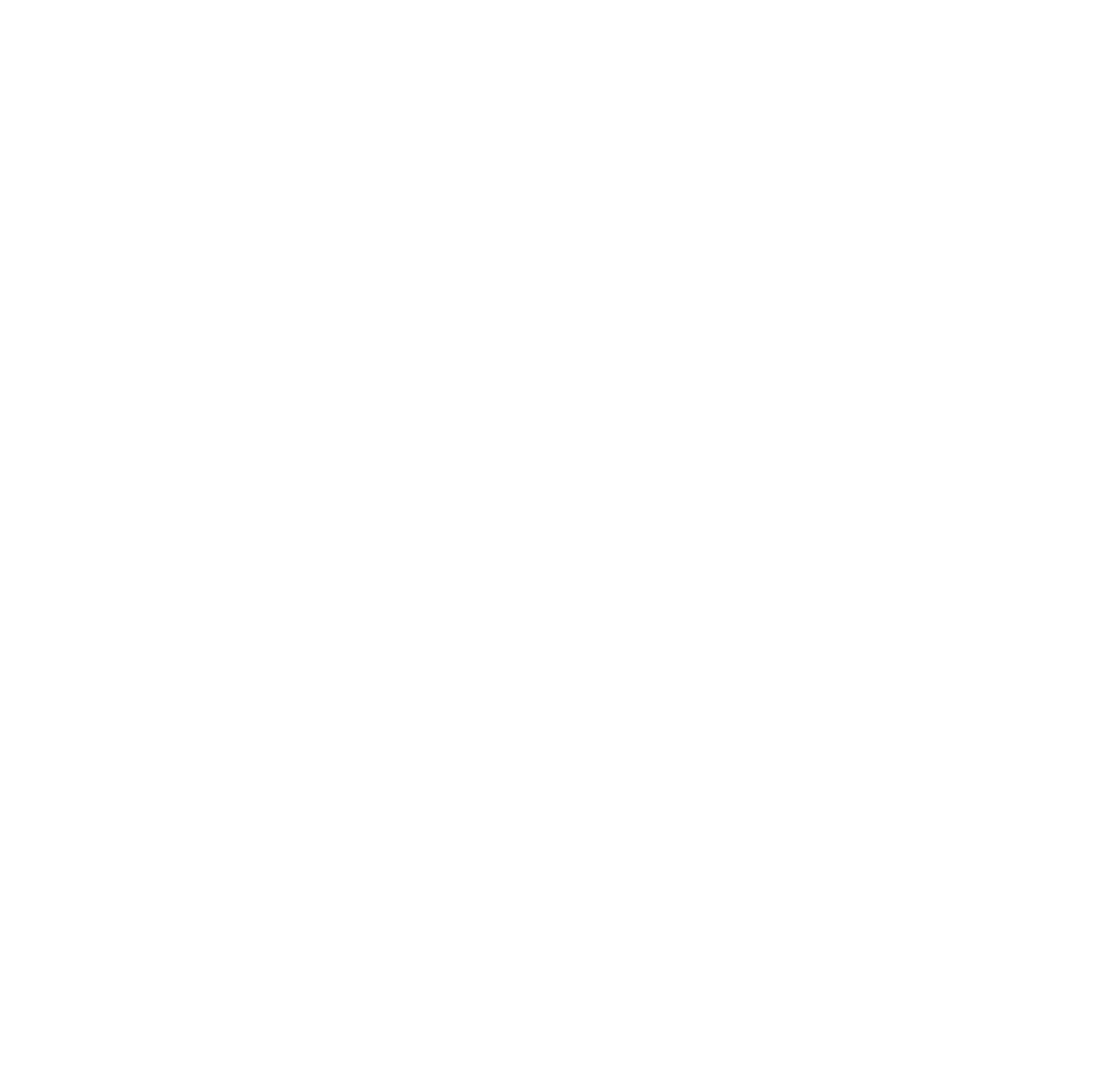

